\title{
Reconfiguration of Traffic Grooming Optical Networks *
}

\author{
Ruhiyyih Mahalati and Rudra Dutta \\ Department of Computer Science, North Carolina State University, Raleigh, NC 27695-7534
}

\begin{abstract}
Advances in optical data transmission and optical signal routing has caused wide expectation for optical networks to form tomorrow's backbone transport. One attractive feature of these networks is the ability to reconfigure the logical topology of the network seen by higher layers with comparative ease and speed by reconfiguring optical switches, without the need to modify the physical topology of the network. On the other hand, with the current mismatch of bandwidth available from individual wavelength channels and typical bandwidth demands, it is also widely recognized that grooming of subwavelength traffic into the full-wavelength channels is an indispensable component of optical network design. The topic of reconfiguration in optical networks that carry subwavelength traffic has received comparatively little attention. In this paper, we consider this problem. Our main contributions are as follows. We discuss the common basis on which grooming effectiveness and reconfiguration efficiency can be considered, and develop a reconfiguration cost function in keeping with this consideration. We formulate the joint problem of reconfiguration and grooming precisely, and offer a heuristic as well as an exact solution method to solve this problem. In offering numerical simulation results for our algorithms, we make the important observation that a disjoint sequential consideration of the two problems leads to solutions that are very inefficient in the joint sense.
\end{abstract}

\section{Introduction}

With recent advances in fiber optics, including Wavelength Division Multiplexing (WDM) and Wavelength Routing, optical networking is widely expected to be the key to satisfying the ever-increasing bandwidth and QoS demand of backbone networks, and to form tomorrow's wide area networks. Signals on different wavelength channels can be independently switched entirely in the optical domain by optical switch fabrics, allowing network operators to form lightpaths, i.e. clear end-to-end optical channels. The set of

*This research was supported in part by NSF grant \# ANI-0322107 such lightpaths formed is called a virtual or logical topology. One of the most attractive features of such a paradigm is the ability to reconfigure the logical topology on demand, e.g. when the traffic demands change. Such reconfiguration is accomplished by reconfiguring the switching (optical or electronic) state of intermediate node (thus modifying the logical topology as needed), and rerouting traffic flow over the lightpaths of the new virtual topology.

Current optical technology allows each wavelength channel to provide a bandwidth of $10 \mathrm{Gbps}$, while $40 \mathrm{Gbps}$ channels are on the horizon, while individual traffic demand components vary in bandwidth and are typically of much lower rate. To carry such traffic efficiently over an optical network, many individual traffic components have to be aggregated for transport over wavelength channels, giving rise to a multiplexing problem. At intermediate nodes the optical signals have to be terminated into electronic equipment such as Digital Cross-Connects (DXCs) capable of separating the traffic components for separate routing. This not only requires expensive electronic equipment, but breaks the clear optical channels, increasing delay for the traffic and otherwise offsetting the advantages of optical transmission. The design of strategies to carry subwavelength traffic components, integrated with logical topology design, to minimize electronic processing or required electronic equipment at intermediate nodes, has become an important research area known as traffic grooming in the last few years.

Prior Work : Given the importance of these research areas, it is not surprising that significant work exists in literature. Recent years have seen a large number of studies on traffic grooming, including some comprehensive surveys of literature [5]. Most of these focus on some heuristic design algorithms, since the grooming problem is known to be NPComplete [4]. Some authors attempt to apply generic solution methods such as genetic algorithms $[2,7,16]$, others bring domain-specific insights to bear on the design problem $[12,13,15]$. In most cases, the design goal is the minimization of some measure of the network cost such as the number of Line Terminating Equipment (LTE), or the electronic processing load, at intermediate nodes. The cost may 
be added over all network nodes, or at the node where this cost is maximum [3]. Recently there has been some work on articulating a dynamic version of the problem; we have discussed this area in more detail in [8].

The performance measures of interest for reconfiguration studies are: (i) Disruption to traffic currently being carried, or network availability during reconfiguration, and (ii) Time from when reconfiguration is initiated till it is complete. Early interest in reconfiguration of optical networks was focused on broadcast optical LANs [1,9]. Elemental reconfiguration operations such as two-branch exchanges [9] were defined which can be combined to perform complete reconfigurations with an eye to minimize the disruption aspect. A survey of reconfiguration issues arising in broadcast optical networks is available in [1], which also formulates the problem as a Markov decision process. More recently, attention has shifted to wavelength routed wide area networks and the reconfiguration issues involved. With most reasonable cost models, the reconfiguration problem is also computationally hard. Heuristics have been proposed that try to minimize the disruption to the network by making small elemental changes to the virtual topology at each step $[6,11]$.

However, until this time, no attention has been paid in the literature to a joint consideration of these problems, to the best of our knowledge. This is the topic of our research presented in this paper. Next we briefly discuss the motivation for this research.

A Need for Joint Design : It is well known that a disjoint and independent consideration of two (or more) aspects of a problem usually results in overall suboptimal design, even if the individual subsolutions used in the various phases are good or even optimal. In optimizational terms, the forced sequential consideration of the subproblems effects an inexact decomposition of the complete problem, introducing artificial constraints that reduce the space of achievable solutions, possibly leaving most of even all of the globally optimal solutions out of reach of such an approach. Nevertheless, such disjoint approaches are very popular in design, because of the conceptual simplicity and tractability they afford; also in many cases the suboptimality involved is negligible.

It might be assumed that the reconfiguration problem in traffic grooming networks is one where a disjoint approach is sufficient. Reconfiguration could thus proceed without any awareness that subwavelength traffic existed in the network, and existing full-wavelength reconfiguration strategies could be used without change. The primary value of the research we present here is that it shows such a strategy, while attractively simple, is inadequate by reasonable standards. Independently operating grooming and reconfiguration strategies, however good in themselves, are not likely to work well together. A reconfiguration strategy that seeks to minimize the number of reconfiguration steps, for example, may dictate a final virtual topology that results in very high OEO switching (transparent to the reconfiguration algorithm). Our main contributions are as follows. First we discuss a common basis on which the quality of a grooming solution and a reconfiguration strategy can be jointly considered. This allows us to define a meaningful cost metric for reconfiguration, and integrate it with a traditional grooming objective. We formulate the integrated problem as an ILP, and also develop a heuristic approach to solving the integrated problem. Finally, we provide results from numerical simulations that show the validity of our conclusions.

The rest of this paper is arranged as follows. In the next section, we define the problem precisely, first discussing and defining the reconfiguration cost function. In Section 3 we present our heuristic approach. Section 4 contains our numerical results, and Section 5 concludes the paper.

\section{Problem Definition}

To develop a common basis for considering grooming and reconfiguration related costs, we consider the optical and electronic switches at the intermediate nodes which are the equipment that physically implement both the grooming and the reconfiguration. Typically, the backbone networks comprise of a number of OXCs arranged in some arbitrary topology, connected by directed optical fiber link. Each OXC can optically switch a signal from a particular input port (a fiber link) to a particular output port, independent of the other wavelengths. The switching of the same wavelength at successive OXCs implements a directed lightpath. Conceptually, the channels on each wavelength are isolated from the optical signals on each incoming fiber and independently switched to outgoing fibers. At each outgoing fiber, the signals on different wavelength are re-multiplexed onto the fiber link. DXCs are deployed along with OXCs to enable grooming by extracting and electronically switching individual lower-speed streams aggregated inside lightpaths. We model each node to possess a monolithic DXC with limited capacity (i.e., the node does not have the capability to switch all traffic on all wavelengths of all incoming fibers simultaneously). A monolithic DXC has a single switching fabric that is capable of switching TDM slots on any of the wavelengths from its input ports to any other wavelength on any output port. To enable some traffic to be switched at the DXC, the lightpath carrying that traffic must be terminated optically, all traffic on that lightpath must be converted to electronic form, and back to the optical after the DXC stage. This is referred to as Optical-ElectricalOptical (OEO) conversion, which is undesirable due to the reasons mentioned earlier. 
An instance of the traffic grooming problem is given by a physical topology and a traffic matrix $T$. Conceptually, a feasible solution to the grooming problem can be considered to comprise of the following three components: (i) The virtual topology to be implemented, (ii) Routing and Wavelength Assignment (RWA) for each lightpath in the virtual topology, and (iii) Routing of each traffic component in the traffic matrix on the lightpaths. We denote the virtual topology with RWA solution by $\mathcal{T}$, and the mapping of subwavelength traffic on the virtual topology $\mathcal{T}$ by $\mathcal{G}$. We focus on the fine-grained grooming cost model as in [4], then the cost of a particular solution is given by the total OEO switching performed in the solution $\mathcal{G}$.

When the offered traffic to the network changes, the grooming cost may rise if the network continues to operate with $\mathcal{T}$ and $\mathcal{G}$. This cost can be avoided by reconfiguring the OXCs and DXCs, to implement a new grooming solution. A grooming gain is obtained in terms of the reduced amount of electronic switching required by the new solution under the changed traffic conditions. The grooming cost calculated in terms of total amount of electronic switching models the total traffic weighted delay in the network, since OEO conversion introduces delay. On the other hand, we usually perceive the reconfiguration cost in terms of the number of OXCs and DXCs that need to be reconfigured (this is in keeping with both the concerns of disruption to traffic and time taken to complete the reconfiguration, as mentioned above). Every time an OXC or a DXC needs to be reconfigured, the traffic being switched through them is delayed. Thus, the reconfiguration cost models the total delay experienced by the traffic at the nodes that need to be reconfigured. This provides the common basis on which to combine and compare grooming and reconfiguration costs.

However, the issue of relative importance remains to be determined. We see this as a network architecture and design goal related decision, and not a general quantity we can fix for all networks. The grooming cost is a constant operating cost to the network while the reconfiguration cost is a transient cost incurred only at the time of reconfiguration. A badly groomed network will continue to incur a high grooming cost until either the network conditions change again or reconfiguration is undertaken. On the other hand, a bad reconfiguration strategy would result in a high reconfiguration cost only at the time of reconfiguring the network. Thus, to be able to reflect the grooming gain effectively in comparison to the reconfiguration cost we multiply it by a factor of $\gamma$. Therefore $\gamma$ is related to the average delay between reconfigurations. On the same lines, we allow a transition from the old to a new grooming solution only when the grooming gain is at least $\delta$ more than the reconfiguration cost, thus, avoiding "flapping". The proper value for each of these parameters $\gamma$ and $\delta$ are highly dependent on many network specific factors, such as the physical topology, traf- fic pattern being supported by the network, operating policy etc. It is upto the network administrator to determine the values of these parameters based on the characteristics of the network, and it is beyond the scope of this work. In Section 4, we present our experience with various values of these parameters.

In developing the integrated cost function from the above considerations, we make the following assumptions:

- Wavelength continuity constraint is assumed to hold, i.e. a lightpath must be assigned the same wavelength on all the links it traverses. From a practical perspective, this means we assume wavelength converters are absent in the network.

- Bifurcated routing of traffic [5] is not allowed and the total traffic for any source-destination $(s-d)$ pair is at the most equal to the capacity $C$ of a wavelength (i.e. $\left.t^{(s d)} \leq C\right)$. Disallowing bifurcated routing implies that the entire traffic $\left(t^{(s d)}\right)$ for a particular $s$ - $d$ pair follows the same lightpath or sequence of lightpaths from source to destination.

- No more than a single lightpath is allowed between any s-d node pair.

The last two assumptions are made to simplify the reconfiguration cost development. While our approach can be adopted for both these situations, we choose to proceed with the simplifying assumptions instead of focusing on these complexities which are not central to our research. We have studied the case of multiple lightpaths between s-d nodes pairs in [10].

Notations : We use notation generally consistent with [5]. We consider an arbitrary physical topology of $N$ nodes, with the nodes numbered from 0 to $N-1$. There is either one directed fiber link from a node $l$ to any other node $m$, or none. At any given time, the solution components $\mathcal{T}$ and $\mathcal{G}$ are static. When the aggregate traffic changes beyond some significant amount over a long time scale (hours or days), the problem is to find a different virtual topology $\mathcal{T}^{\prime}$ as well as a final groomed solution $\mathcal{G}^{\prime}$.

In naming the parameters and variables, the endpoints of a physical link are denoted by $l, m$. The source and destination of the traffic are denoted by $s, d$; while $i, j$ denote the endpoints of a lightpath. The lightpath indicator $b_{i j}$ is a binary variable that denotes whether a lightpath exits from node $i$ to $j$, while $b_{i j}(l, m)$ indicates if such a lightpath traverses the physical link from node $l$ to $m$. The link indicator $p_{l m}$ is 1 if a physical link exists between nodes $l$ and $m$. The traffic indicator $d_{i j}^{(s d)}$ is 1 if the lightpath from $i$ to $j$ carries traffic from source $s$ to destination $d$. The linklightpath-wavelength indicator $c_{i j}^{(k)}(l, m)$ is 1 if a lightpath 
from node $i$ to $j$ on wavelength $k$ traverses the physical link from node $l$ to $m$. The traffic demands between pairs of nodes in the physical topology are specified as the traffic matrix $T=\left[t^{(s d)}\right]$. We assume that the network supports traffic streams at rates that are a multiple of some basic rate (e.g., OC-3). We let $C$ denote the capacity of each wavelength expressed in units of this basic rate. Each quantity $t^{(s d)} \in\{0,1,2, \cdots, C\}$ is also expressed in terms of the basic rate, and it denotes the number of traffic units that originate at node $s$ and terminate at node $d$. The lightpath from node $i$ to $j$ carries aggregate traffic $t_{i j}$, and $t_{i j}^{(s d)}$ denotes the amount of this traffic which is due to the demand $t^{(s d)}$. Thus, a virtual topology solution $\mathcal{T}$ is specified by values for variables $c_{i j}^{(k)}(l, m), b_{i j}$ and $b_{i j}(l, m)$; a groomed solution $\mathcal{G}$ is given by values for $d_{i j}^{(s d)}, t^{(s d)}$ and $t_{i j}^{(s d)}$.

\subsection{Defining the Integrated Cost Function}

We describe below a procedure for reconfiguration cost calculation by obtaining matrix representations for the switching state of each node in the old and new topology and grooming solutions, and then calculating the distance between these two matrices in order to determine the number of OXC and DXC changes. Clearly, different reconfiguration strategies will incur different costs for the same reconfiguration depending on their specifics; e.g. a reconfiguration strategy that reconfigures different OXCs in parallel will require less time in general than another strategy which reconfigures them serially. Similarly, different OXC fabrics have different actual implementations [14], which determine what reconfigurations can be performed on the switch simultaneously, or how much reconfiguration effort a given reconfiguration of the switch should be modelled as. Our methodology can be adapted to reflect specific switch fabric implementations. However, for any particular strategy, the basic approach we define below can be reasonably expected to be very useful, because the number of OXC and DXC reconfigurations affect the cost in a very real sense. We have considered some other definitions of the global cost that are also likely to be practically useful, details can be found in [10].

Matrix Representation of Each Node: The switching state of each node $n$ is represented by a $W \times N_{s}$ matrix, where $N_{s}$ represents the total number of OXC inputs and $W$ is the total number of wavelengths supported by each fiber. Of the $N_{s}$ inputs, the first $N_{f}$ represent incoming optical fibers for node $n$, and the rest represent DXC inputs/outputs per wavelength. The switching state of a node $n$ is determined only by non-zero $c_{i j}^{(k)}(l, m)$ related to that node, and the state is represented by the following symbols.

- Electronic Termination: Electronic termination of a

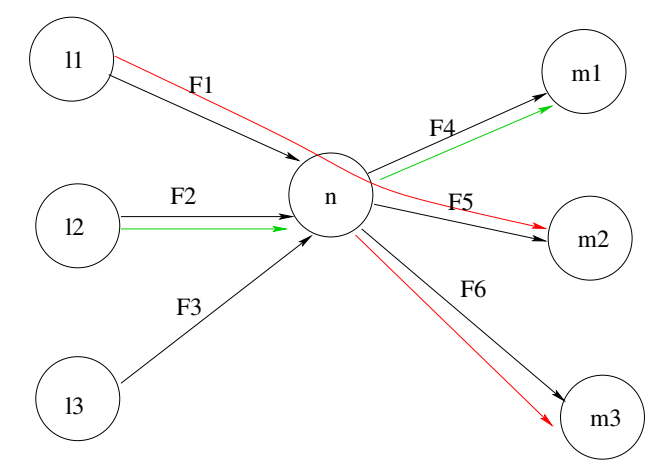

Figure 1. Physical and virtual connectivity, node $n$

lightpath at node $n$ on wavelength $k$ is indicated by an indicator with $(n=m=j)$ and is represented by symbol ' $\mathrm{O}$ ' in the matrix at row $k$ and column $f_{i}$.

- Electronic Generation: Origination of a lightpath is indicated by an indicator with $(n=l=i)$ and is represented by a fiber number $f_{o}$ in one of the columns from $N_{f}+1$ to $N_{s}$ in row $k$.

- Optical Switching: An optical switching of a lightpath at a node $n$ is indicated by $((n=m) \neq j \neq i)$. We represent this by a fiber number $f_{o}$ in one of the columns from 1 to $N_{f}$ in row $k$. A fiber number $f_{o}$ in column $f_{i}, 1 \leq f_{i} \leq N_{f}$, represents that the traffic on wavelength $k$ is switched from the fiber $f_{i}$ to fiber $f_{o}$.

- Don't Care: It is represented by symbol ' $\mathrm{X}$ ' in the matrix meaning there is no traffic on that fiber's wavelength for node $n$. It does not matter if a lightpath is established or not.

Consider the example shown in Figure 1. Let the solid red line represent wavelength $\lambda_{1}$, while the dotted green line represents wavelength $\lambda_{2}$. Then the matrix representation for node $n$ is:

\begin{tabular}{|c|c|c|c|c|c|}
\hline & $F 1$ & $F 2$ & $F 3$ & $d x c 1$ & $d x c 2$ \\
\hline$\lambda_{1}$ & $F 5$ & $X$ & $X$ & $F 6$ & $X$ \\
\hline$\lambda_{2}$ & $X$ & $O$ & $X$ & $F 4$ & $X$ \\
\hline
\end{tabular}

where $d x c 1$ and $d x c 2$ represent the DXC inputs/outputs (assuming two DXC inputs/outputs per wavelength).

Distance Calculation between Matrices : Given the matrix representation of a node $n$ in both $(\mathcal{T}, \mathcal{G})$ and $\left(\mathcal{T}^{\prime}, \mathcal{G}^{\prime}\right)$, we determine the distance between these two matrices in terms of whether the OXC/DXC need to be reconfigured or not. We make the following observation: both the OXC and 
the DXC will require reconfiguration if a new lightpath (absent in the old virtual topology) is being established or an old lightpath has been eliminated. The OXC has to be reconfigured because a wavelength channel has to be switched to/from DXC port, and the DXC because it has to switch the tributary traffic components in the wavelength channel. On the other hand, a change in the optical switching of a lightpath in the new virtual topology when compared with the old topology requires only the OXC to be reconfigured. However, if new lightpaths are terminated and originated at node $n$ on the same wavelength, which was not the case in the old topology, then it requires a single reconfiguration change to both the OXC and the DXC. For example, if the lightpath of wavelength $\lambda_{1}$ passing through node $n$ were to be electronically terminated and originated at node $n$ due to a reconfiguration, the relevant row in the matrix representation would change to:

$$
\lambda_{1} \quad\left(\begin{array}{ccccc}
F 1 & F 2 & F 3 & d x c 1 & d x c 2 \\
O & X & X & F 6 & F 5
\end{array}\right)
$$

We reasonably assume that a change from any state to a Don't Care state does not require any reconfiguration effort. This is because when the state of a node for a lightpath changes to Don't Care it means no one is receiving the data from that lightpath. But every state change from a Don't Care to any other state must now be assumed to require reconfiguration; since we cannot assume anything about the state of the node before it became Don't Care.

Global Reconfiguration Cost Calculation Methods : As mentioned above, the DXC at each node is a monolithic DXC with limited capacity. Electronic termination and electronic generation result in the same amount of reconfiguration effort. That is, the reconfiguration of the DXC is binary in nature represented by $i s_{-} d x c_{-}$recon $f i g_{n}$, incurring a total cost of $\beta$.

In our cost calculation method we consider a cost of $\alpha_{1}$ for each OXC reconfiguration i.e. some cost is incurred for every change on each wavelength. This is a suitable method for reconfiguration cost calculation when all the changes in the new virtual topology are implemented individually irrespective of the other changes. The variable used to indicate the total number of changes required by an $\mathrm{OXC}$ at node $n$ is $o_{2} \_r c_{-} c o u n t_{n}$. The value of $o x c_{-} r c_{-}$count $_{n}$ is the summation of all the non zero elements of the distance matrix for node $n$ that requires reconfiguration of the $\mathrm{OXC}$, as specified above. The total reconfiguration cost is given by the following equation.

$$
R C=\alpha_{1} \sum_{n=0}^{N-1} o_{0} c_{-} r c_{-} \text {count } t_{n}+\beta \sum_{n=0}^{N-1} i s_{-} d x c_{-} r c_{n}
$$

Linearizing Reconfiguration Cost : Though the above discussed method involving distance matrix accurately cap- tures reconfiguration cost, it is non-linear in nature and cannot be implemented using any integer linear optimization solver. Here we formulate a nearly equivalent linear function which we use in our CPLEX implementation to prove the correctness and efficiency of our proposed ILP and benchmark the performance of our heuristic against optimal results.

In this cost function we count every change in the matrix representations that needs the OXC and/or DXC to be reconfigured as a separate change incurring cost. We assign a cost of $\alpha_{2}$ for every OXC reconfiguration and cost $\beta_{2}$ for every DXC reconfiguration. We use the $c_{i j}^{(k) \prime}(l, m)$ and $c_{i j}^{(k)}(l, m)$ values to calculate the cost, these are the linklightpath-wavelength indicators from the new and old solutions respectively. Based on the rules discussed in Section 2.1, we formulate the following linear equation to calculate the total number of electronic generations in the new topology that were absent in the old topology.

$$
\begin{aligned}
r 1 & =\sum_{j, k, m}\left(c_{n j}^{(k) \prime}(n, m)-c_{n j}^{(k)}(n, m)\right) \\
& -\left(\left(c_{n j}^{(k) \prime}(n, m)-c_{n j}^{(k)}(n, m)\right) c_{n j}^{(k)}(n, m)\right) \forall n
\end{aligned}
$$

The linear equation 3 represents the total number of electronic terminations in the new topology that were absent in the old topology.

$$
\begin{aligned}
r 2 & =\sum_{i, k, l}\left(c_{i n}^{(k) \prime}(l, n)-c_{i n}^{(k)}(l, n)\right) \\
& -\left(\left(c_{i n}^{(k) \prime}(l, n)-c_{i n}^{(k)}(l, n)\right) c_{i n}^{(k)}(l, n)\right) \forall n
\end{aligned}
$$

The linear equations 4 and 5 represent the total number of optical switchings which have changed, i.e. these equations captures all those cases where a lightpath which was being optically switched in the old topology is still being optically switched but on a different fiber in the new topology. It also counts all those cases where a lightpath which was being electronically generated or terminated or both in the old topology is now being optically switched.

$$
\begin{aligned}
r 3 & =\sum_{k, l, m, i \neq n, j \neq n}\left(c_{i j}^{(k) \prime}(l, n)-c_{i j}^{(k)}(l, n)\right) \\
& -\left(\left(c_{i j}^{(k) \prime}(l, n)-c_{i j}^{(k)}(l, n)\right) c_{i j}^{(k)}(l, n)\right) \forall n \\
r 4= & \sum_{k, l, m, i \neq n, j \neq n}\left(c_{i j}^{(k) \prime}(n, m)-c_{i j}^{(k)}(n, m)\right) \\
& -\left(\left(c_{i j}^{(k) \prime}(n, m)-c_{i j}^{(k)}(n, m)\right) c_{i j}^{(k)}(n, m)\right) \forall n
\end{aligned}
$$

The total reconfiguration cost is given by the following linear equation.

$$
\begin{aligned}
R C_{L i n} & =\phi\left(\left\{c_{i j}^{(k)}(l, m)\right\},\left\{c_{i j}^{(k) \prime}(l, m)\right\}\right) \\
= & (r 1+r 2+r 3+r 4) \alpha_{2}+(r 1+r 2) \beta_{2}
\end{aligned}
$$

However, the "if-then" nature of the OXC/DXC reconfiguration cost calculation that we illustrated above (when 
a single pass-through lightpath is electronically terminated at an intermediate node) is fundamentally non-linearizable. As a consequence, $R C_{L i n}$ considers a lightpath termination and generation at a node $n$ on a particular wavelength as two separate changes to both the OXC and the DXC, as opposed to a single change considered by $R C . R C_{\text {Lin }}$ considers every change that requires DXC reconfiguration as a separate change, thus making a slight overestimate and changing the binary nature of DXC reconfiguration.

In the above, we have used parameters $\alpha$ and $\beta$ for the sake of generality. These parameters can be used to weigh the cost in favor of OXC reconfiguration versus DXC reconfiguration, or vice versa. If there is no reason to favor either, they should be set equal (perhaps to 1); this is what we do in our numerical simulations, as presented in Section 4.

It remains to compute the gain in grooming cost that can be obtained by undertaking the reconfiguration from an old solution give by $\mathcal{T}$ and $\mathcal{G}$ to a new solution given by $\mathcal{T}^{\prime}$ and $\mathcal{G}^{\prime}$. With our earlier definitions of grooming cost, the grooming gain is easily seen to be:

$$
\begin{aligned}
& \psi\left(\left\{d_{i j}^{(s d)}\right\},\left\{d_{i j}^{(s d)} \prime\right\},\left\{t^{(s d)}\right\}\right)= \\
& \left(\sum_{s, d, i, j} d_{i j}^{(s d)} t^{(s d)}-\sum_{s, d} t^{(s d)}\right) \\
& -\left(\sum_{s, d, i, j} d_{i j}^{(s d) \prime} t^{(s d)}-\sum_{s, d} t^{(s d)}\right)
\end{aligned}
$$

where the primed versions of the individual variables refer to the new solution. In the above, we have reasonably assumed that when the offered traffic changes, the grooming cost must be calculated on the basis of the existing mapping of the traffic to the virtual topology, but with the magnitudes of the changed traffic currently offered.

It is now straightforward to define the entire problem as an ILP, following the ILP of [5]. Additional parameters must be added to the formulation (to represent the old traffic matrix, and existing solutions $\mathcal{T}$ and $\mathcal{G}$. New constraints are also needed to enforce the additional assumptions we made above, as well as to adopt the new variables $d_{i j}^{(s d)}$ defined above. However, the significant difference from existing ILPs lies in the integrated cost function, which is defined as:

$$
\begin{aligned}
\psi\left(\left\{d_{i j}^{(s d) \prime}\right\}\right. & \left.,\left\{d_{i j}^{(s d)}\right\},\left\{t^{(s d)}\right\}\right) \gamma \\
- & \phi\left(\left\{x_{i j}^{k}(l, m)\right\},\left\{c_{i j}^{(k)}(l, m)\right\}\right)-\delta
\end{aligned}
$$

Briefly, the above cost function, to be maximized, specifies that the new grooming solution to be found must maximize the difference between grooming gain in adopting that solution and the reconfiguration cost incurred in doing so. We have discussed the role of the scale factor $\gamma$ in Section 2. The parameter $\delta$, also discussed above, serves to forbid any new solution adoption unless a minimum net gain is achieved, thus increasing the stability of the solution. A complete specification of the ILP, as well as more discussion on the grooming cost gain calculation, may be found in [10]. They are omitted here for the sake of brevity.

\section{Heuristic Approach}

While such an ILP can be used in theory to solve the integrated problem, in practice it is too time consuming to compute it on a frequent basis for any but the smallest networks. Apart from this, it makes no proactive attempts to choose a solution that is likely to be proof against future changes in traffic. Clearly, such considerations could be introduced in the formulation, but only at the cost of making it even harder to solve. Realistically, we recognize that when traffic has changed drastically from the original pattern, this computational effort may be appropriate, but a practical means to react swiftly to more moderate (and probably, more frequent) changes to traffic is desired.

A practical strategy to address those concerns must have the following goals. The heuristic approach must (i) avoid resorting to the full ILP whenever possible, (ii) ward off failure of the network, that is a situation in which any increase in the traffic components would cause the current grooming solution $\mathcal{G}$ to fail to carry the new traffic, (iii) avoid adopting grooming solutions that are very much suboptimal, in decreasing order of importance. The rationale is as follows: the first concern must be to minimize ILP use, otherwise this heuristic may become equivalent to simply running the ILP each time. Secondly, we know that if any feasible solutions to the changed traffic conditions exist, the ILP approach would find the optimal such feasible solution. However, feasibility is more attainable than optimality, so by avoiding the ILP, we must lower our sights to at least remain feasible. Since the heuristic will run repeatedly on the same network (with successively changing traffic patterns), the feasibility concern is represented by the next goal, which makes the heuristic somewhat proactive in seeking continued feasibility. Finally, we still want to address the grooming cost concern, even though it has to be subjugated to the first two needs. Briefly, the second and third goals describe the reconfiguration and the grooming goals respectively.

Since the problem is intractable in nature, a tractable heuristic is unlikely to attain globally optimal solutions. For this reason, we attempt to apply heuristic methods when it appears that the new traffic matrix represents a local change in traffic, in some sense. When the reach of the change in traffic is close to global, the heuristic simply indicates that the ILP solution approach should be employed.

\subsection{Over-Provisioning of Traffic Matrices}

We use overprovisioning as a proactive measure to protect the grooming solution from failure. Specifically, given a groomed solution $\mathcal{G}$, we apply a modified version of this solution, $\mathcal{G}_{o p}$ to the actual network. In $\mathcal{G}_{o p}$, some or all traffic elements are increased by some amount such that 
the network is made to carry as much traffic as possible on all the lightpaths without violating the capacity constraint. This method increases the probability of a new traffic matrix being supported on the existing virtual topology, as we demonstrate below. Overprovisioning thus adds dummy traffic to the network without affecting the cost of the grooming solution. However, a real increase in traffic in the future can be accommodated by carrying it in place of some of the dummy traffic, and in such a case the actual grooming cost would increase.

To see that overprovisioning helps in continued feasibility of a grooming solution, consider the family of traffic matrices that a given grooming solution $\mathcal{G}$ can feasibly carry over the chosen virtual topology $\mathcal{T}$. It is obvious that any decrease in any traffic component will always retain feasiblity (although the mapping of the traffic on the lightpaths might not be optimal with respect to the grooming cost). It is equally obvious that any increase in any traffic component will cause failure in the grooming solution even if the spare capacity exists in the lightpaths of $\mathcal{T}$ to carry it, because the DXCs at the source, destination, and intermediate nodes for that traffic component will not be configured to switch the extra TDM slots corresponding to the increase. If the DXCs had originally been configured to switch dummy TDM slots together with the real traffic components to utilize the spare capacity, any increase of traffic upto the spare capacity could have been accommodated. In general, of two grooming solutions which differ only in some spare capacity on some lightpath that is (1) left unassigned by one solution $\mathcal{G}_{1}$, and (2) assigned as over-provisioning to one or more traffic elements traversing that lightpath by another solution $\mathcal{G}_{2}$, the latter solution supports a strictly larger superset of changed traffic matrices than the former one.

We perform the overprovisioning iteratively. Since we are unlikely to have estimates of which traffic components are more likely to increase in the future, in each iteration we allocate the following equal amount of over-provisioned traffic to each $t_{i j}^{(s d)}$ on each lightpath from $i$ to $j$ that it traverses, with $s_{i j}$ being the total number of sub-wavelength elements on that particular lightpath.

$$
t_{-} o_{i j}^{(s d)}=\left\{\begin{array}{lr}
\left(C-\sum_{s, d} t_{i j}^{(s d)}\right) / s_{i j}, & t^{(s d)} \neq 0 \\
0, & \text { otherwise }
\end{array}\right\} .
$$

This is a fair allocation method, as each sub-wavelength element is over-provisioned by the same amount, irrespective of the actual amount of traffic carried by them. The iterative character of our algorithm is motivated by the observation that traffic from a particular source to destination may flow over many lightpaths. The amount of over-provisioned traffic $t_{-} o_{i j}^{(s d)}$ as calculated above may be different on different lightpaths for the same traffic element $t_{i j}^{(s d)}$. The maximum amount of over-provisioned traffic that we can actually assign to a particular $s-d$ pair is the minimum of all the overprovisioned values calculated on all the lightpaths where the traffic for this $s$-d pair flows; this guarantees that the capacity constraint is not violated on any of the lightpaths. However, simply using this minimum for each $t_{i j}^{(s d)}$ may leave unused slack on many lightpaths because the initial allocation on each lightpath was equal, an iterative approach can allocate this slack by noting which traffic components have already been overprovisioned and are not in a position to accept any more overprovisioning. Noting that traffic components which flow over many lightpaths are likely to be able to accept the minimum overprovisioning, we formulate the Iterative-Max-Lightpath approach: at each iteration, select the traffic component $t_{l}$ that flows over the maximum number of lightpaths and has not yet been over-provisioned. Overprovision this traffic component by the minimum $t_{\min }$ of the $t_{-} o_{i j}^{(s d)}$ it has currently received, and mark it as already overprovisioned. For each lightpath traversed by $t_{l}$ on which it was assigned a $t_{-} o_{i j}^{(s d)}>t_{\min }$, there was some share of the slack that $t_{l}$ was unable to accept; recompute the $t_{-} o_{i j}^{(s d)}$ values on this lightpath for all traffic components that traverse it, reallocating that share equally. The iteration is continued till all the traffic components have been marked as overprovisioned. At this stage, we have the complete overprovisioned traffic matrix.

We have investigated several other approaches to allocating the the spare capacity. Details can be found in [10]; however, we note that the above approach produces the best overprovisioning over a large range of instances, and for this reason we have chosen to present it above as well as use it for our numerical simulations as presented in Section 4.

\subsection{Reconfiguration Heuristic}

In this section we propose a reconfiguration heuristic, which is designed with the three goals mentioned at the beginning of Section 3 in mind. We show the entire flow of the reconfiguration heuristic in Figure 2, and explain the important points below.

The reconfiguration heuristic is triggered for every change in the traffic matrix. Given a physical topology, an initial traffic matrix $T$ and a new traffic matrix $T_{\text {new }}$, we obtain an optimal groomed solution $\mathcal{G}$ by solving the corresponding ILP. We then calculate the over-provisioned traffic matrix as above, denoted by $T_{\text {prov }}=\left[t_{\text {prov }}^{(s d)}\right]$. In order to determine if the new traffic would require the virtual topology to be reconfigured or not, we calculate the difference matrix $T_{\text {diff }}$ given by the following equation.

$$
t_{\text {diff }}^{(s d)}=t_{\text {prov }}^{(s d)}-t_{\text {new }}^{(s d)} \forall s, d
$$

We do not reconfigure any network component unless any of the $T_{\text {diff }}$ matrix elements touch the threshold value. 
The threshold value is a limit that we use to determine the $s-d$ pairs that are carrying traffic very close to the current over-provisioned traffic. The value of this threshold limit can be determined by the network administrator based on the general characteristics of the input traffic pattern. Any difference matrix $T_{\text {diff }}$ element which touches the threshold value, falls in the critical region. We perform reconfiguration whenever any traffic element falls in the critical region, to bring the elements below the threshold limit.

In choosing the reconfiguration method, we attempt to distinguish between local and global changes. Specifically, we create two criteria calling for reconfiguration, based on the number of affected lightpaths, i.e. lightpaths which carry at least one traffic component that is in its critical region.

Hard Decision Criterion : when more than a given fraction LPlimit of the lightpaths of $\mathcal{T}$ are affected. We can reasonably expect this criterion to be met less frequently. The traffic change is likely to be of global scope, and the appropriate response is to solve the integrated ILP. In situations when this criterion is met but the integrated ILP results in a negative objective value, in a real-life scenario it would be appropriate to issue an alert message to the network administrator, informing him/her that many traffic demands are in the critical region but no reconfiguration can be performed cost effectively. This could act as a trigger for the administrator/ operator to consider deploying more network resources. If the ILP finds an optimal solution, the network is reconfigured to that solution.

Soft Decision Criterion : when less than LPlimit of the lightpaths of $\mathcal{T}$ are affected. The traffic change is probably local in nature. Accordingly, we attempt the following three reconfigurations of local but sucessively larger scope. We stop with whichever reconfiguration succeeds in reducing the number of traffic components in the critical region. If none of them succeed, the appropriate action is to alert the network operator as before. We note that all three reconfigurations only involve DXCs and do not change the state of the OXCs, in keeping with our desire to undertake only low cost reconfigurations prompted by local traffic changes. More detailed description of these algorithms can be found in [10].

Recalculation of Over-Provisioned Traffic Matrix : The over-provisioned traffic matrix is recalculated with the aim of increasing the over-provisioned share of those subwavelength elements which are in the critical region, either by utilizing any extra capacity on the lightpaths or by reducing the over-provisioned share of other sub-wavelength elements that are not in the critical region. We use two flavors of this method successively: in the first, we only include the affected lightpaths; in the second flavor we also consider lightpaths that carry traffic components that also traverse affected lightpaths. It is easy to show that there are cases in which the second approach will successfully re-overprovision the capacity, while the first fails. The second approach is thus more general, but it generally results in more reconfiguration.

Rerouting Traffic in the Critical Region : In this method of local reconfiguration, we try to reroute all the subwavelength elements that are in the critical region. We select all the sub-wavelength elements that are in the critical region, then we use Dijkstra's algorithm to find an alternate route for these sub-wavelength elements on the existing lightpaths. When determining the alternate route for a sub-wavelength element, we make sure that the capacity constraint is not violated on any of the lightpaths in the alternate route.

Rerouting the Entire Traffic: If the above local reconfiguration methods fail to result in a new over-provisioned traffic matrix where the total number of sub-wavelength elements that are in the critical region are reduced, we try rerouting all the sub-wavelength elements on the lightpaths of $\mathcal{T}$. This method reroutes traffic by solving an ILP that minimizes the total traffic carried by the lightpath which carries the maximum traffic. This has the effect of maximizing slack on lightpaths, making it possible for the overprovisioning algorithm to find a good allocation if such allocations exist. This method obviously is computationally costly (though much less than solving the integrated ILP), and in addition requires rearrangement of traffic not directly affected by the changed traffic pattern.

\section{Numerical Results}

For the purpose of numerical simulations, the reconfiguration heuristic was coded in $\mathrm{C} / \mathrm{C}++$. The exact solutions to the grooming approach and the integrated approach were coded as ILPs using ILOG CPLEX 7.1, a software designed to solve linear optimization problems. However, due to the complexity and the intractable nature of the problem, each instance that we ran with CPLEX took from a few minutes to several hours. For larger instances CPLEX took several days to give optimum results, hence for the larger part of our experiments we have used physical topologies of 6 nodes. We used four different physical topologies: a unidirectional ring, a bidirectional path, and two topologies (which we refer to as "dumbbell" and "barbell") which both consist of two strongly connected sets of three nodes, the two sets connected weakly. We generated traffic matrices randomly with uniform distribution. Randomly generated matrices that turned out to be infeasible on the chosen topology were discarded. Each traffic matrix was subjected to an evolution of 20 changes. In each change, each traffic component was modified by a randomly generated amount, uniformly distributed between 0 and $C$.

Our results for the four different topologies were very 
similar. Figures 3,4,5 show some of our representative results. We plot the cumulative value of the integrated objective for three distinct approaches to reconfiguration in response to the traffic evolution. The first is the completely disjoint approach we mentioned in Section 1. For each traffic change, the new optimal grooming solution is calculated, and the network is reconfigured accordingly. We naturally expect this approach to perform badly with respect to the integrated cost. The second approach is to solve the ILP for each new traffic matrix. Naturally, this is the best strategy, but as we know it is prohibitively computation intensive. The third approach is the heuristic one we presented above. The cumulation of the cost metric allows us to see the effect over time when the network is reconfigured following the various approaches.

As we can see, the disjoint approach using grooming only continues to incur high reconfiguration cost and thus the integrated objective continues to grow worse over time. The ILP performs well as expected, and defines the limit of achievable benefit from any approach. Finally, we see that the heuristic achieves a much better performance over time than the disjoint approach, even though only a small fraction of the computation required for the ILP approach was required (for the case of Figure 3, the ILP was run only for 2 of the 20 traffic changes). If a lower value of LPlimit is used, the computational requirement will grow, but the heuristic will track the ILP approach more closely. We have presented results with the value of LPlimit set to $70 \%$, though our experiments show that values as low as $30 \%$ still succeed in reducing the number of calls to the integrated ILP significantly [10].

\section{Conclusion}

We have considered the problem of reconfiguration of wavelength routed optical networks in the context of groomed subwavelength traffic. We have shown that the problem requires an integrated approach, and articulated a heuristic algorithm as well as a precise formulation of the problem. Our numerical results show that the heuristic approach performs well with reasonable computation demands.

There are many ways in which this research can be extended, and we are pursuing some of them. In particular, we believe that the overprovisioning method can be extended to have wider application. We are also investigating the issue of reducing the ILP computation further, without losing solution quality.

\section{References}

[1] I. Baldine and G. Rouskas. Traffic adaptive wdm networks: a study of reconfiguration issues. Journal-of-Lightwave-
Technology, 19(4):433-55, 2001.

[2] R. Berry and E. Modiano. Reducing electronic multiplexing costs in SONET/WDM rings with dynamically changing traffic. IEEE Journal on Selected Areas in Communications, 18(10):1961-1971, Oct 2000.

[3] B. Chen, G. Rouskas, and R. Dutta. Traffic grooming in WDM unidirectional paths and rings with min-max cost objective. In Proceedings of Networking 2004, Athens, Greece, pages 174-185, May 2004.

[4] R. Dutta, S. Huang, and G. Rouskas. On optimal traffic grooming in elemental network topologies. In Proceedings of OPTICOMM, Dallas, Texas, pages 13-24, October 2003.

[5] R. Dutta and G. Rouskas. Traffic grooming in WDM networks: Past and future. IEEE Network, 16(6):46-56, Nov/Dec 2002.

[6] A. Gencata and B. Mukherjee. Virtual-topology adaptation for wdm mesh networks under dynamic traffic. In Proceedings of IEEE INFOCOM, volume 1, pages 48-56, 2002.

[7] O. Gerstel, R. Ramaswami, and G. Sasaki. Cost effective traffic grooming in WDM rings. IEEE/ACM Transactions on Networking, 8(5):618-630, Oct 2000.

[8] S. Huang and R. Dutta. Research problems in dynamic traffic grooming in optical networks. In Proceedings of First International Workshop on Traffic Grooming, San Jose, October, 2004, 2004.

[9] J.-F. Labourdette, G. Hart, and A. Acampora. Branchexchange sequences for reconfiguration of lightwave networks. IEEE/ACM Transactions on Communications, 42(10):2822-32, Oct 1994.

[10] R. Mahalati. Reconfiguration of sub-wavelength groomed wavelength routed optical networks. Master's thesis, North Carolina State University, 2003. Publicly available via WWW: http://www.lib.ncsu.edu/ETD-db/.

[11] A. Narula-Tam and E. Modiano. Dynamic load balancing in wdm packet networks with and without wavelength constraints. IEEE Journal on Selected Areas in Communications, 18(10):1972-9, Oct 2000.

[12] J. Simmons, E. Goldstein, and A. Saleh. Quantifying the benefit of wavelength add-drop in WDM rings with distance-independent and dependent traffic. Journal of Lightwave Technology, pages 48-57, Jan 1999.

[13] P.-J. Wan, G. Calinescu, L. Liu, , and O. Frieder. Grooming of arbitrary traffic in SONET/WDM BLSRs. IEEE Journal on Selected Areas in Communications, 18(10):1995-2003, Oct 2000.

[14] Y. Wang and X. Chen. Comprehensive analysis of different optical interconnecting architectures. In Proceedings of the SPIE The International Society for Optical Engineering, volume 4582, pages 205-215, 2001.

[15] X. Zhang and C. Qiao. On scheduling all-to-all personalized connections and cost-effective designs in WDM rings. IEEE/ACM Transactions on Networking, 7(3):435-445, Jun 1999.

[16] K. Zhu and B. Mukherjee. Traffic grooming in an optical WDM mesh network. IEEE Journal on Selected Areas in Communications, 20(1):122 -133, Jan 2002. 


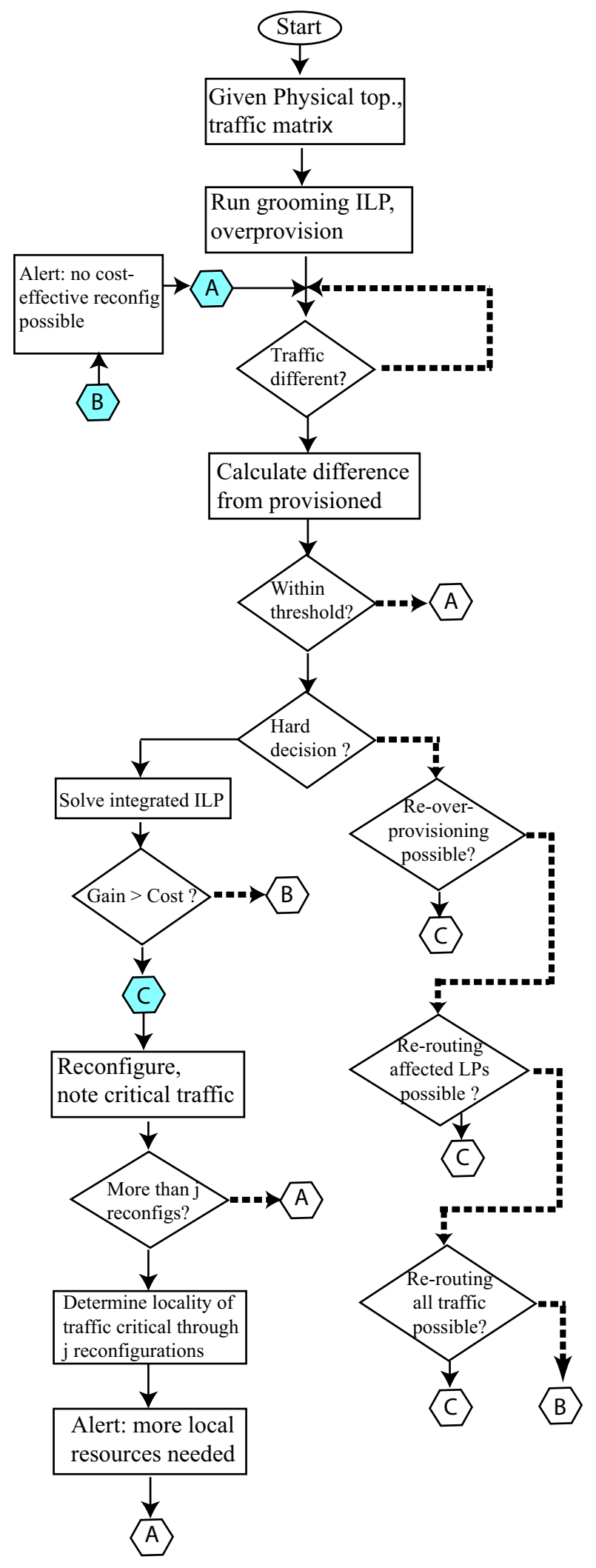

Figure 2. Heuristic Approach

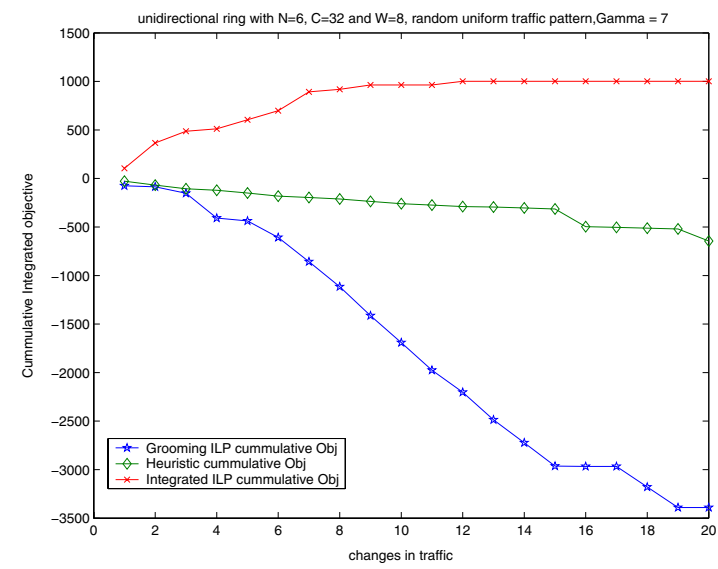

Figure 3. Cumulative objective function for unidirectional ring

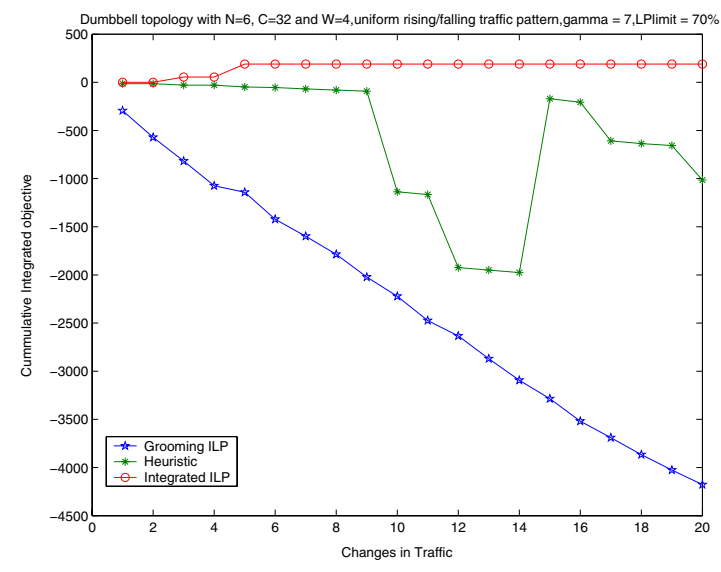

Figure 4. Cumulative objective function for dumbbell

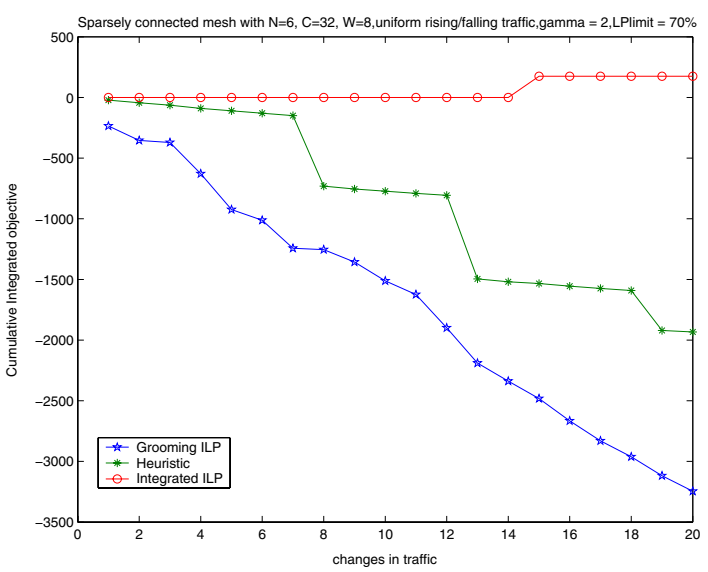

Figure 5. Cumulative objective function for barbell 